\title{
Isoflavonoide in der Behandlung der Hautalterung postmenopausaler Frauen
}

\section{Bayerl \\ D. Keil}

\author{
Isoflavonoids in the Treatment of Skin Aging in Postmenopausal Women
}

\section{Zusammenfassung}

Präventive und kurative Maßnahmen zur Verzögerung der Hautalterung haben bei Frauen in fortgeschrittenen Lebensjahren einen besonderen Stellenwert. Phytoöstrogen-haltige Cremes bieten postmenopausalen Frauen ein wirksames sowie zugleich verträgliches und sicheres Instrument, um der hormonbedingten Hautalterung entgegenzutreten. Dies belegt eine kontrollierte Multizenterstudie an 234 Probandinnen.

\section{Abstract}

Preventive and therapeutic measures to retard skin aging in women are of outmost importance. Cremes containing phytoestrogens are convenient and safe tools to work against hormonal skin aging in postmenopausal women. This has been shown by a controlled multi-center study in 234 volunteers.

\section{Einleitung}

Mit fortschreitender Zahl der Lebensjahre wird die Haut atrophisch, weniger elastisch, trocken und zunehmend faltig. Kleinere Blutgefäße werden vermehrt sichtbar, die Thermoregulation der Haut lässt nach, der Hautstoffwechsel wird schwächer und die Wundheilung erfolgt verlangsamt. Viele dieser hauteigenen Alterungsprozesse werden hormonell reguliert und der rapide Östrogenabfall in der Menopause trägt maßgeblich zur intrinsischen hormonellen Hautalterung bei [16].

Eine Hormonsubstitution bietet postmenopausalen Frauen die Möglichkeit, diesen endogenen Hormonmangel auszugleichen. Die zahlreichen positiven Auswirkungen einer Östrogentherapie bei postmenopausalen Frauen sind gut bekannt. Wie man seit einiger Zeit weiß, besitzen systemisch applizierte Östrogene neben der Wirkung auf die klimakterischen Symptome auch sehr positive Effekte auf die Hautalterung $[2,10]$. Durch verschiedene Un- tersuchungen und epidemiologische Studien wurde belegt, dass topisch applizierte Östrogene [14]:

- zu einer besseren Organisation der elastischen Fasern führen

- den Gehalt an Kollagen Typ III erhöhen

- die Dicke der Haut steigern

- die Elastizität verbessern

- die Trockenheit reduzieren

- die Faltenintensität vermindern.

Trotz dieses nachgewiesenen Nutzens auf klimakterische Symptome und die Hautalterung wird eine Hormonersatztherapie zur Zeit aufgrund der ersten Ergebnisse aus der „Women Health Initiative" mit der Auswertung einer großen amerikanischen epidemiologischen Studie zur Hormonersatztherapie bei Frauen kontrovers diskutiert [5,11]. Viele Frauen lehnen eine systemische Hormonsubstitution aufgrund der potenziellen Risiken hinsichtlich Ovarialkarzinomen, Myokardinfarkt, Thrombosen und Apoplex ab. Eine topische Applikation von Östrogenen erscheint hinsichtlich der positiven Wirkung auf die Hautalterung vielver-

Institutsangaben

Klinik für Dermatologie, Venerologie und Allergologie Klinikum Mannheim gGmbH (Direktor: Prof. Dr. S. Goerdt), Universitätsklinikum, Fakultät für Klinische Medizin Mannheim der Universität Heidelberg

Korrespondenzadresse

PD Dr. med. Christiane Bayerl · Klinik für Dermatologie, Venerologie und Allergologie · Universitätsklinikum ·

Theodor-Kutzer-Ufer 1-3·68135 Mannheim·E-mail: christiane.bayerl@haut.ma.uni-heidelberg.de

Bibliografie

Akt Dermatol 2002; 28: S14-S18 @ Georg Thieme Verlag Stuttgart · New York · ISSN 0340-2541 
sprechend [14], doch aufgrund der gesetzlichen Bestimmungen dürfen kosmetischen Produkten keine Hormone zugesetzt werden (Quelle: $\S 1$ in Verbindung mit Anlage 1 Nr. 260 der Kosmetik-Verordnung).

\section{Phytoöstrogene: Östrogenähnliche Wirkung} aus dem Reich der Natur

Phytoöstrogene bieten Frauen eine Alternative, die sich neben der guten Wirksamkeit durch eine sehr gute Verträglichkeit und Sicherheit auszeichnet. Phytoöstrogene finden sich in einer Vielzahl von pflanzlichen Lebensmitteln und werden täglich mit der Nahrung aufgenommen. Die östrogenähnliche Wirkung von bestimmten Pflanzen wurde 1926 erstmals beschrieben [6]. Bis 1975 wurden mehrere hundert Pflanzen mit östrogenähnlicher Wirkung identifiziert [3].

Phytoöstrogene werden in die drei Gruppen Isoflavone, Lignane und Coumestane unterteilt. Isoflavone sind in tropischen Früchten, in Kaffee, in Artischocken und in Trauben enthalten (Abb.1). Der höchste Gehalt von Isoflavonen befindet sich in Sojabohnen. Die hauptsächlichen Bestandteile der Isoflavone sind Genistein und Daidzein. Diese nicht-steroidalen Moleküle besitzen aufgrund der strukturellen und funktionellen Homologie mit 17- $\beta$-Östradiol eine östrogenähnliche Wirkung (Abb.2). Vermutet wird derzeit, dass Phytoöstrogene ähnlich wie die Östrogene mit dem Östrogenrezeptor interagieren [13,17].

Epidemiologischen Studien zufolge ist der Konsum phytoöstrogenhaltiger Nahrungsmittel ernährungsphysiologisch empfehlenswert. So sind westliche Zivilisationskrankheiten in Asien und anderen Ländern mit einer sojareichen Ernährung deutlich geringer $[4,12]$. Insbesondere hormonabhängige Tumoren wie Brust-, Prostata- und Ovarialkrebs sowie kardiovaskuläre Erkrankungen treten aufgrund der besonderen Ernährungsgewohnheiten in diesen Ländern seltener auf [8].

Nach topischer Applikation auf die postmenopausale Haut bewirken Phytoöstrogene eine Proliferation der Epidermis sowie eine Synthese von Kollagen. Zugleich schützen die Phytoöstrogene das Kollagen vor der enzymatischen Degradation [10]. Seit neuestem werden Phytoöstrogene in Anti-Aging-Hautcremes eingesetzt. Das Präparat (Novadiol, Vichy-Cosmetique Active Deutschland), das die beiden Soja-Isoflavonoide Genistein und Daidzein enthält, soll die Hautalterung postmenopausaler Frauen gezielt verlangsamen. Nach regelmäßiger Applikation der Intensivpflege Novadiol nimmt die Epidermisdicke im Vergleich zur unbehandelten Haut deutlich zu, wie histologische Untersuchungen belegen [9] (Abb. 3).

Nach transdermaler Absorption reichern sich die beiden Isoflavonoide in der Haut an, ohne sich in höheren Konzentrationen im Organismus zu verteilen. Über den Urin ausgeschieden werden lediglich [15]:

- 15,9\% Daidzein nach der ersten Applikation

- 1,6\% Daidzein nach der zweiten Applikation sowie

- 7,7\% Genistein nach der ersten Applikation

- $0,7 \%$ Genistein nach der zweiten Applikation.

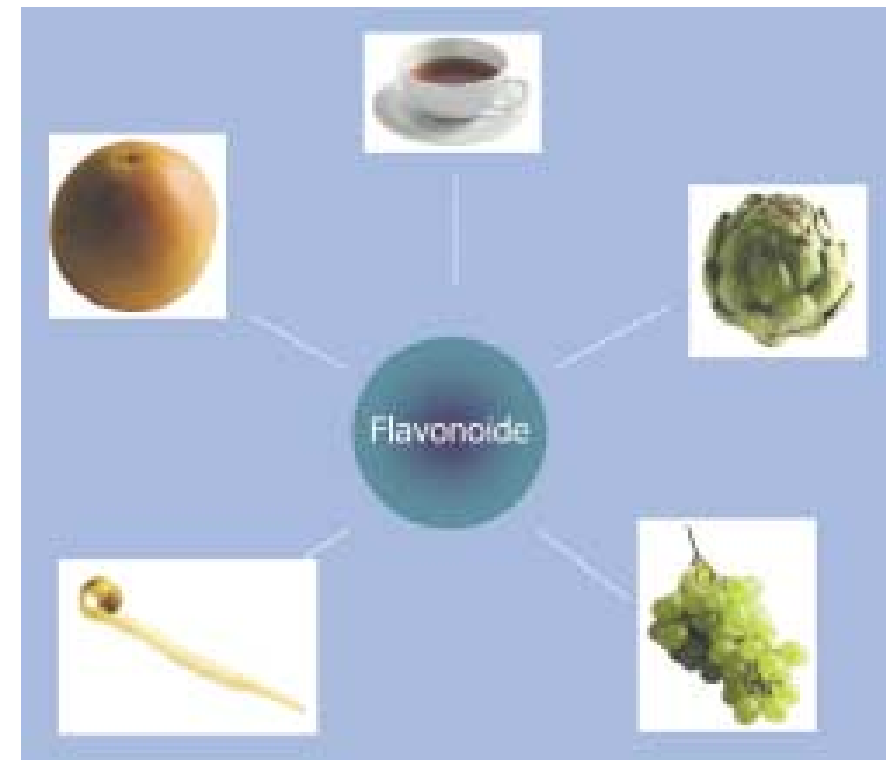

Abb. 1 Phytoöstrogene stammen aus Pflanzen und werden täglich mit der Nahrung aufgenommen.
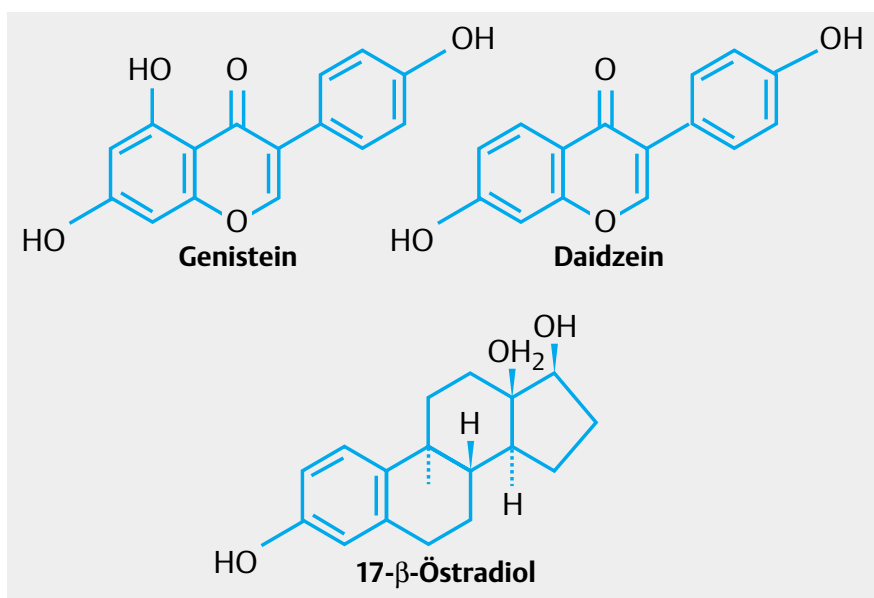

Abb. 2 Phytoöstrogene sind den Östrogenen in der Struktur sehr ähnlich.

Die biologische Potenz der Isoflavonoide ist deutlich geringer als bei synthetischen Östrogenen, wie Bioassays humaner Zellkulturen belegen. Definiert man die biologische Potenz der Östrogene mit 100, liegt Genistein bei 0,084 und Daidzein bei 0,013 [7]. Demnach sind systemische Nebenwirkungen nach topischer Applikation unwahrscheinlich.

\section{Studiendesign}

Die Wirksamkeit einer isoflavonhaltigen Intensivpflege wurde im Rahmen einer kontrollierten, offenen Multizenterstudie an acht verschiedenen europäischen Kliniken in Deutschland, Frankreich, Italien, der Schweiz und in Spanien untersucht. An der Studie nahmen 234 Frauen (Typ der Lichtempfindlichkeit II bis IV) mit einer mindestens drei Jahre zurückliegenden Menopause im Alter von maximal 65 Jahren teil. Die Studienteilneh- 

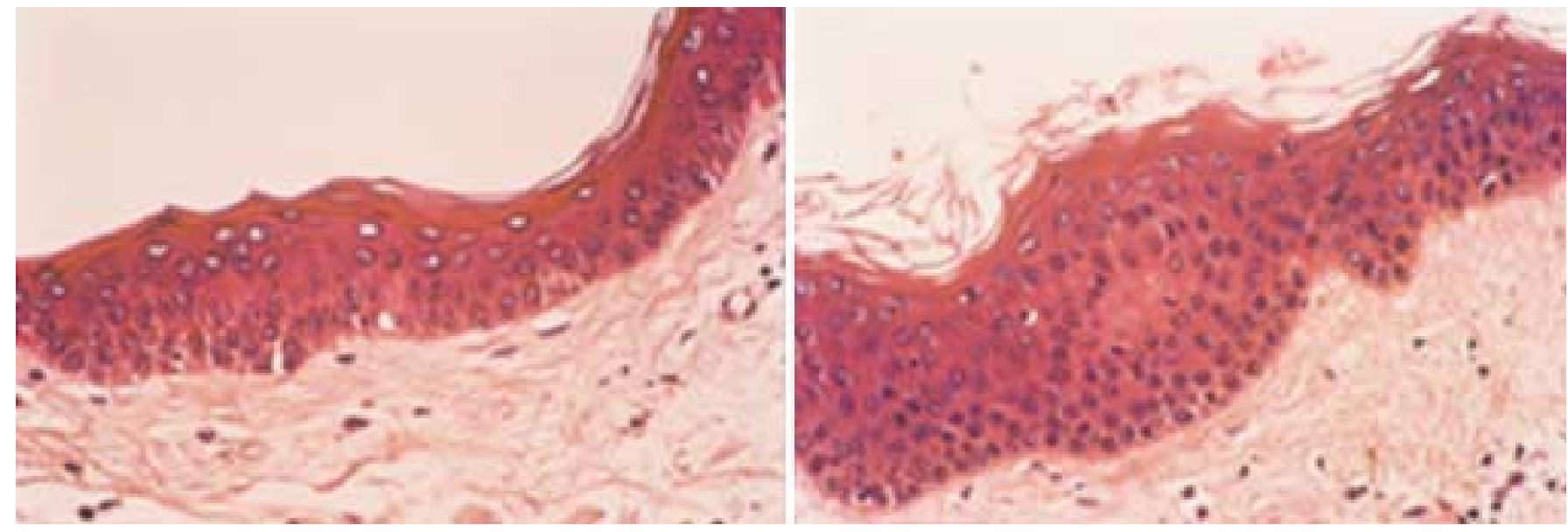

Abb. 3 Histologische Untersuchungen belegen, dass die Epidermisdicke nach regelmäßiger Applikation von Novadiol im Vergleich zur unbehandelten Haut deutlich zunimmt.
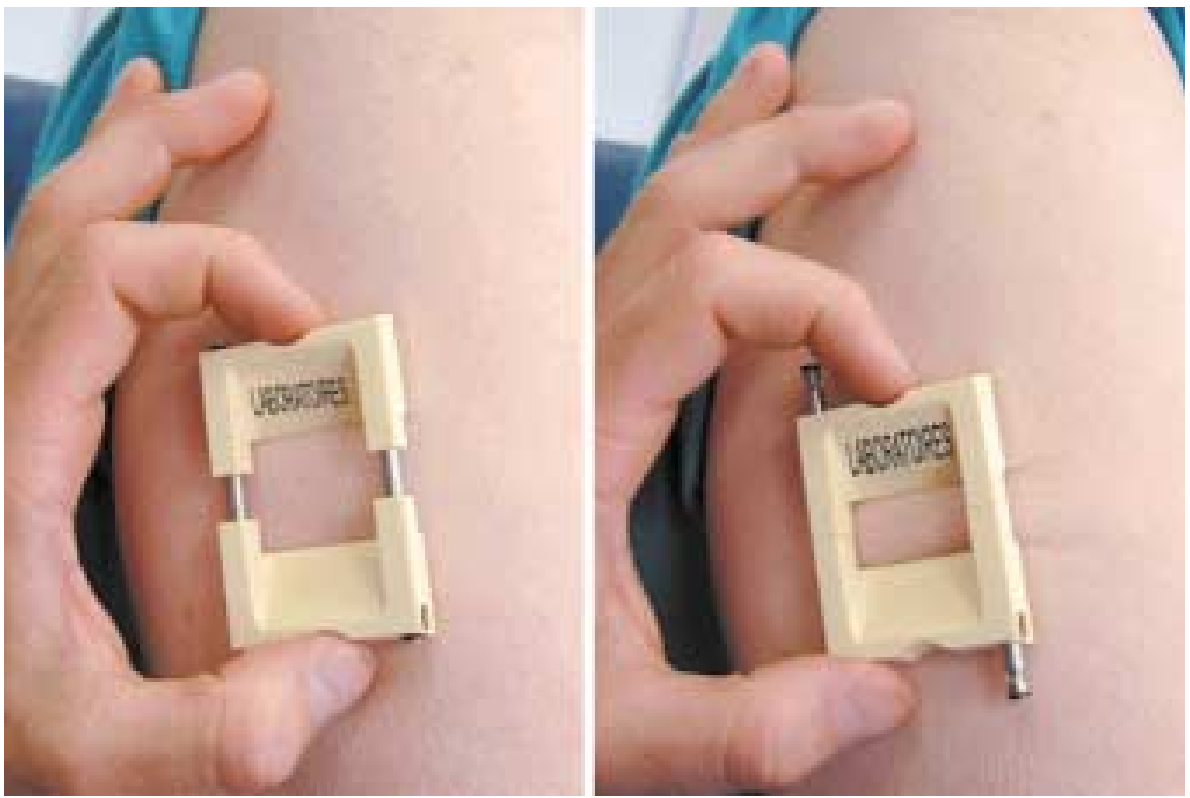

Abb. 4 Der DensiScore ${ }^{\circledR}$ ermöglicht eine instrumentelle Messung des Faltenscores.

merinnen wurden nicht mit einer Hormonersatztherapie behandelt und hatten in den vergangenen drei Monaten keine retinol-, alpha-hydroxy-acids- oder phytoöstrogenhaltige Präparate benutzt. Die topische Applikation der isoflavonhaltigen Intensivpflege (Novadiol, Vichy-Cosmetique Active Deutschland) erfolgte über 12 Wochen zweimal täglich auf das Gesicht, den Hals und jeweils einen Oberarm. Der andere Arm blieb unbehandelt und diente als Kontrolle.

\section{Messgrößen/Beurteilungskriterien}

Die Auswertung basiert auf den klinisch-dermatologisch erhobenen, objektivierbaren Befunden mit einem semiquantitativen Messgerät. Die Intensität der Hautfalten wurde unter dermatologischer Kontrolle mit Hilfe des DensiScore ${ }^{\circledR}$ ermittelt (Abb. 4) [1] Hierbei wird ein durch das Gerät definiertes Hautareal mit gleich bleibendem horizontalen Druck zusammen geschoben. Das im Messfeld entstehende Faltenprofil wird anschließend mit einem sechsstufigen Referenzatlas verglichen und in eine von sechs Ka- tegorien von Grad 1 (junge Haut) bis Grad 6 (gealterte Haut) eingestuft (Abb.5). Während jüngere Haut sich in geringerem Ausmaß und vergleichsweise regelmäßig faltet, charakterisieren ältere Haut tiefe und ungleichmäßige Falten.

Zusätzlich wurden klinisch durch den Arzt die Trockenheit, die Rauheit, die Falten und die Atonie der Haut zu Studienbeginn sowie nach vier bzw. nach 12 Wochen evaluiert (Skala 0 bis 9). Darüber hinaus hatten die Probandinnen die Möglichkeit, eine Beurteilung abzugeben.

\section{Ergebnisse}

Der mit dem DensiScore ${ }^{\circledR}$ gemessene Faltenscore verbesserte sich bei dem behandelten im Vergleich zu dem unbehandelten Arm ebenfalls signifikant um durchschnittlich $17 \%(p<0,05)$ (Abb. 6). 


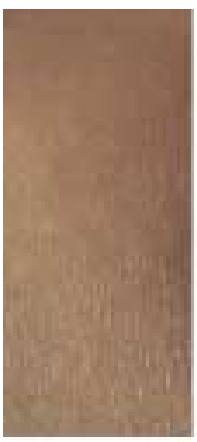

1

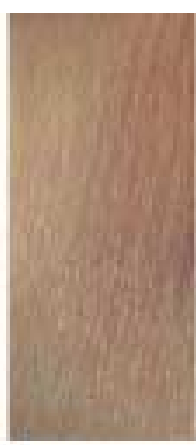

2

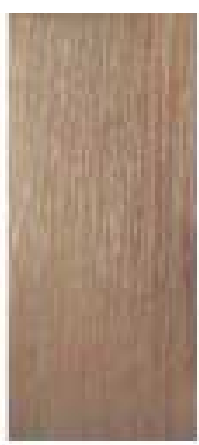

3

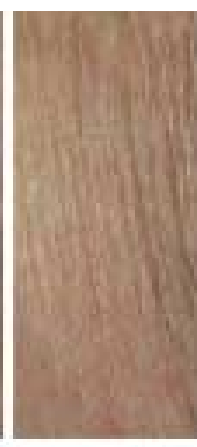

4

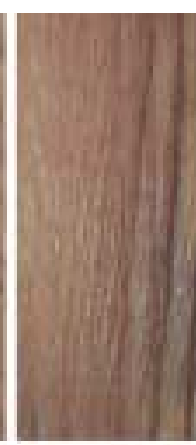

5

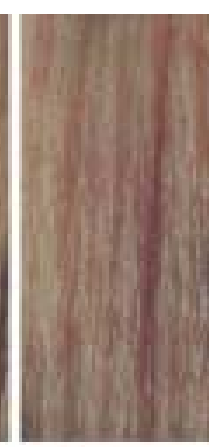

6
Abb. 5 Das Faltenprofil wird mit einem sechsstufigen Referenzatlas verglichen. Feine regelmäßige Faltenbildung bei junger Haut (Stufe1) und verstärkt auftretende Falten und Faltentiefe bei älterer Haut (Stufe 6).

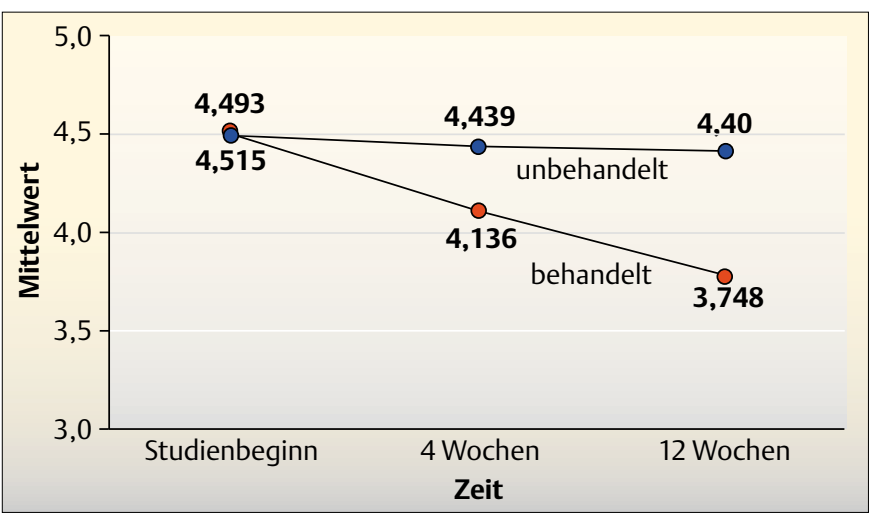

Abb. 6 Der Faltenscore bestätigt einen deutlichen Unterschied zwischen dem behandelten und dem unbehandelten Arm $(p<0,05)$.

Bereits nach den ersten vier Behandlungswochen kam es zu einer qualitativen Verbesserung des Hautbildes, die sich kontinuierlich bis zum Studienende fortsetzte. Nach 12 Wochen waren die Symptome der Hautalterung statistisch signifikant vermindert. Die Beurteilung durch den Studienarzt zeigte, dass die Haut deutlich weniger trocken, weniger rau ist, eine höhere Tonizität hatte und weniger Falten festgestellt wurden (Tab.1, Abb. 7):

- Am deutlichsten war die positive Wirkung auf die Trockenheit der Gesichtshaut ausgeprägt. Diese verbesserte sich nach 12 Wochen um bis zu 32,9\%.

- Parallel dazu verminderte sich die Hautrauigkeit signifikant um bis zu $22 \%(\mathrm{p}<0,05)$.

- Gesichtsfalten wurden um $20 \%$ vermindert $(p<0,05)$.

- Die für die postmenopausale Haut typische Atonie ging ebenfalls statistisch signifikant um $24 \%$ zurück $(p<0,05)$.

In Einklang damit steht die Selbstbeurteilung der Probandinnen die der phytoöstrogenhaltigen Creme eine gute Wirkung auf die Haut attestierten (Tab. 2). Bereits nach einer einmonatigen Anwendung bestätigten die Probandinnen, dass sich das Hautbild positiv verändert hatte. Sowohl die Hydratation, die Hautfestigkeit als auch die Ausstrahlung verbesserten sich ihrer Einschätzung zufolge etwa um bis zu der Hälfte (54, 46, $43 \%$ ).
Tab. 1 Klinische Auswertung durch die Studienärzte

\begin{tabular}{|c|c|c|c|}
\hline \multicolumn{2}{|c|}{ bewertete Parameter } & \multirow{2}{*}{$\begin{array}{l}\text { M1 } \\
-\quad 9,4 \%\end{array}$} & \multirow{2}{*}{$\begin{array}{l}\text { M3/MO } \\
-20,5 \%\end{array}$} \\
\hline Gesicht & Falten & & \\
\hline & Rauheit & $-9,0 \%$ & $-21,9 \%$ \\
\hline & Tonizität & $-10,2 \%$ & $-23,7 \%$ \\
\hline & Trockenheit & $-15,3 \%$ & $-32,9 \%$ \\
\hline \multirow[t]{4}{*}{ Hals } & $\begin{array}{l}\text { Gesamtbeurteilung } \\
\text { der Haut }\end{array}$ & $-7,3 \%$ & $-14,6 \%$ \\
\hline & Erschlaffung der Haut & $-6,5 \%$ & $-16,4 \%$ \\
\hline & Falten & $-7,7 \%$ & $-19,8 \%$ \\
\hline & Hautrelief & $-11,1 \%$ & $-22,6 \%$ \\
\hline
\end{tabular}

M0 = Messung zu Studienbeginn; M1 = Messung nach Monat 1; M3 = Messung nach Monat 3.

\section{Beurteilung}

Mit Phytoöstrogenen steht postmenopausalen Frauen ein wirksames, doch zugleich sanftes und sicheres Anti-Aging-Präparat für die reife Haut zur Verfügung. Die Ergebnisse dieser Studie bestätigen unter kontrollierten Bedingungen, dass die isoflavonhaltige Creme das Erscheinungsbild und die Dichte postmenopausaler Haut sehr positiv beeinflusst. Die Falten wurden durch die Isoflavonoide in signifikantem Ausmaß reduziert und das klinische Bild der Haut deutlich verbessert. Neben dem subjektiven Urteil der Probandinnen und der Beurteilung durch den Arzt wurde dies durch eine objektive, instrumentalisierte Messung bestätigt.

Zur Überprüfung der Wirksamkeit und Verträglichkeit kosmetischer Präparate raten wir wie in dieser Studie, instrumentalisierte objektivierbare Messmethoden zu verwenden. Das Studienprojekt hat uns zudem gezeigt, dass es möglich ist, auch Studien aus dem dermato-kosmetischen Bereich multizentrisch und kontrolliert anzulegen.

Die isoflavonhaltige Intensivpflege eröffnet postmenopausalen Frauen somit eine wirkungsvolle Option, um sich vor den endogenen Zeichen der Hautalterung zu schützen bzw. die Zeichen der endogenen Hautalterung zu korrigieren. 

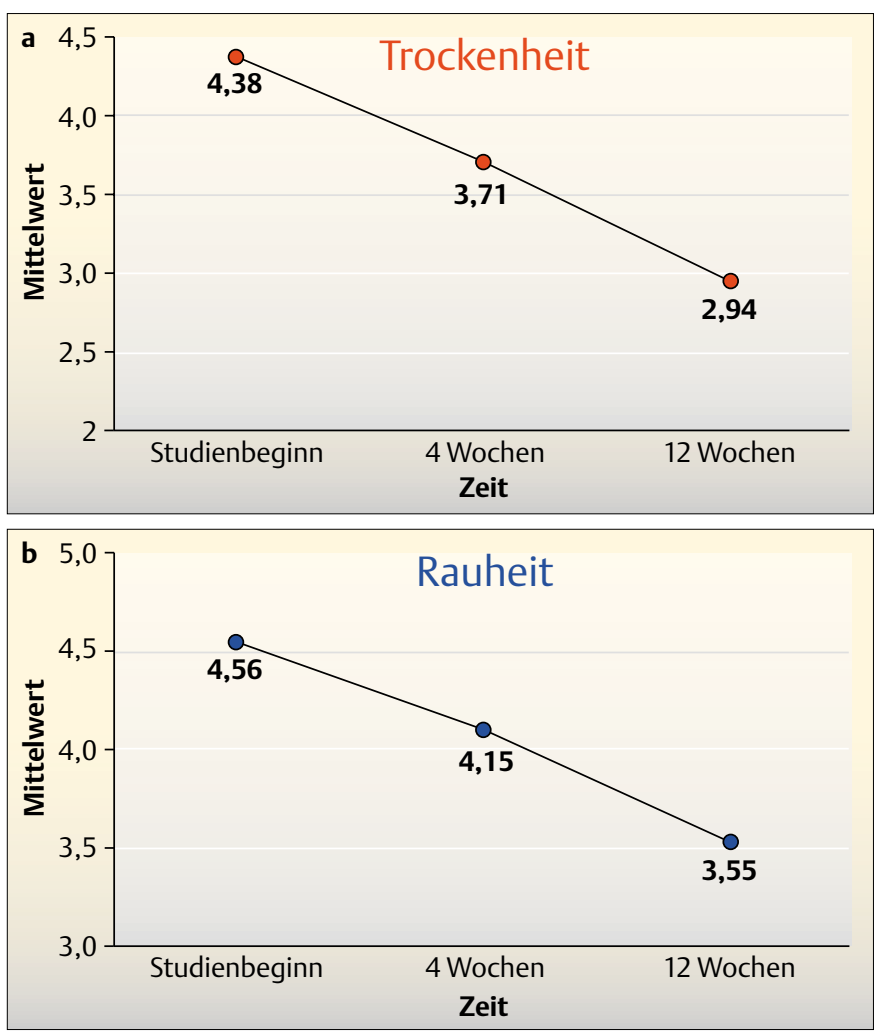

S18
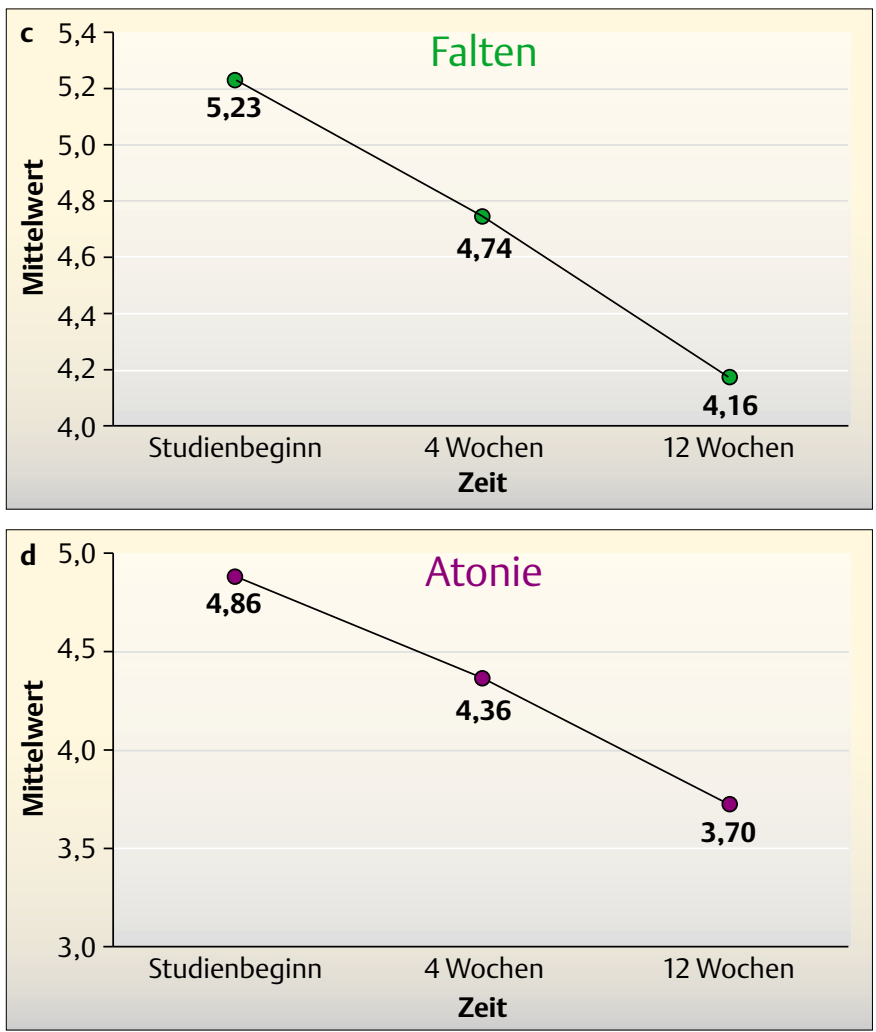

Abb. 7 Innerhalb von 12 Wochen wurde die Trockenheit um 33\% reduziert $(p<0,05)$. Die Rauheit verminderte sich innerhalb des Studienzeitraums um $22 \%(p<0,05)$. Nach der klinischen Evaluation gingen Falten um $20 \%$ zurück $(p<0,05)$. Die Atonie sank durch die Behandlung mit Novadiol um 24\% $(p<0,05)$.
Tab. 2 Selbstbeurteilung der Probandinnen (Anzahl der Probandinnen in \%)

\begin{tabular}{|llll}
\hline \multicolumn{2}{l}{ bewertete Parameter } & M1 & M3/M0 \\
\hline \multirow{2}{*}{ Gesicht } & ausgeprägte Gesichtsfalten & $7,0 \%$ & $12,2 \%$ \\
& mittlere Gesichtsfalten & $11,8 \%$ & $23,3 \%$ \\
\hline & Straffung der Haut & $10,8 \%$ & $28,1 \%$ \\
\hline & Glättung der Haut & $17,5 \%$ & $36,2 \%$ \\
& Prallheit der Haut & $21,4 \%$ & $37,2 \%$ \\
\hline & kleine Gesichtsfältchen & $16,6 \%$ & $37,6 \%$ \\
\hline Hals & Ausstrahlung des Teints & $24,4 \%$ & $43,4 \%$ \\
\hline & Festigkeit der Gesichtshaut & $21,8 \%$ & $45,8 \%$ \\
\hline & Hydratation der Gesichtshaut & $29,7 \%$ & $54,2 \%$ \\
\hline & Falten am Hals & $11,7 \%$ & $28,3 \%$ \\
\hline & Erschlaffung der Haut am Hals & $13,5 \%$ & $29,9 \%$ \\
\hline & Glättung der Haut am Hals & $17,4 \%$ & $32,4 \%$ \\
\hline
\end{tabular}

M0 = Messung zu Studienbeginn; M1 = Messung nach Monat 1; M3 = Messung nach Monat 3.

\section{Literatur}

${ }^{1}$ Bazin R et al. DensiScore ${ }^{\circledR}$ : a new tool for clinical evaluation of age dependent mechanical properties of female skin. Ann Dermatol Venerol 2002; 129: 1S371 - 1S602

${ }^{2}$ Brincat M. Hormone replacement therapy and the skin. Maturitas 2000; 35: 107-117

${ }^{3}$ Farnswoth NR, Bingel AS, Cordell GA, Crane FA, Fong HHS. Potential value of plants as sources of new antifertility agents. J Pharm Sci 1975; 64: $717-754$

${ }^{4}$ Hirayama T. A large-scale cohort study on cancer risks by diet with special reference to the risk reducing effects of green-yellow vegetable consumption. In: Hayashi Y (Ed). Nutrition Cancer. Tokyo: Japanese Scientific Society Press, 1986

${ }^{5}$ Lacey JV Jr, Mink PJ, Lubin JH, Sherman ME, Troisi R, Hertge P, Schatzkin A, Schairer C. Menopausal hormone replacement therapy and risk of ovarial cancer. JAMA 2002; 288: 334-341

${ }^{6}$ Loewe S, Lange F, Spohr E. Über weibliche Sexualhormone. Biochem Zeitschr 1927; 180: 1-26

${ }^{7}$ Markiewitz L, Garey J, Adlerkreutz H, Gurpide E. In vitro bioassay of nonsteroidal phytooestrogens. J Steroid Biochem Molec Biol 1993; 45: 399-405

${ }^{8}$ Messina M. Soy intake and cancer risk: a review of the in vitro and in vivo data. Nutr Cancer 1994; 21: 113 - 131

${ }^{9}$ Noch nicht veröffentlichte Daten von Vichy Cosmetique Active Deutschland.

${ }^{10}$ Punnonen R. Effect of castration and peroral estrogen therapy on the skin. Acta Obstet Gynecol Scand Suppl 1972; 21: 3-44

${ }^{11}$ Rodriguez C, Patel AV, Calle EE, Jacob EJ, Thun MJ. Estrogen replacement therapy and ovarian cancer mortality in a large prospective study of US women. JAMA 2001; 285: 1460-1465

${ }^{12}$ Rose DP, Boyar AP, Wynder EL. International comparisons of mortality rates for breast cancer, ovary, prostate and colon per capital food consumption. Cancer 1986; 58: $2363-2371$

${ }^{13}$ Schmidt JB, Spona J. Steroidrezeptoren in normaler Haut. Z Hautkr 1986; 61: $841-846$

${ }^{14}$ Schmidt JB, Binder M, Demschik G, Biegelmayer C, Reiner A. Treatment of skin aging with topical estrogens. Int J Dermatol 1996; 35: 669-674

15 Vanttinen K, Moravoca J. Transdermal absorption of phytoestrogens. Pharmazie 2001; 56: $711-717$

${ }^{16}$ Varani J, Fisher G, Kang S, Voorhees JJ. Molecular mechanisms of intrinsic skin aging and retinoid-induced repair and reversal. J Invest Dermatol Symp Processings 1998; 3: 57-60

17 Wang TTY, Sathyamoorthy N, Phang JM. Molecular effects of genistein on estrogen receptor mediated pathways. Carcinogenesis 1996; 17: $271-275$ 